\title{
$\Omega$
}

Jana De Brandt' ${ }^{1}$, Sabine Bartel ${ }^{2}$

DI

${ }^{1}$ REVAL - Rehabilitation Research Center, BIOMED - Biomedical Research Institute,

Faculty of Medicine and Life Sciences, Hasselt University, Diepenbeek, Belgium.

2Early Life Origins of Chronic Lung Disease, Research Center Borstel, Leibniz Lung

Center, Member of the German Center for Lung Research (DZL), Borstel, Germany.

\section{Opportunities for early career members at the Lung Science Conference 2019}

\section{Early career forum}

The European Respiratory Society (ERS) Lung Science Conference (LSC) is a yearly conference taking place in Estoril, near Lisbon, Portugal. From March 7 to 10, 2019, around 200 pulmonary researchers and clinicians from all over the world will attend the LSC to discuss the theme of "Mechanisms of acute exacerbation of respiratory disease". Next to the obviously outstanding scientific programme (available from https://cdn. ersnet.org/static/path/documents/programme/ LSC2019WebsiteProgramme090818.pdf), the LSC also offers a lot of opportunities for motivated ERS Early Career Members (ECMs).

First of all, the LSC is a relatively small scientific meeting but with a high density of world-leading experts in their respective fields and members of the ERS Leadership. Thus, it is a great place to network, and to get yourself and your research known to these important people. There are many slots available for posters and oral presentations. Every year, the best five ECM abstracts are chosen and allocated to the Young Investigator Session where the audience chooses the winner of the prestigious William MacNee Award. Please check the webpage for more information (https://www.ersnet.org/congress-and-events/ mechanisms-of-acute-exacerbation-ofrespiratory-disease). ECMs can also apply for travel grants to attend this conference and, if awarded, they will be entitled to a mentorship lunch with selected mentors from the respiratory field.

A highlight for ECMs at the LSC is always the Saturday afternoon career development session (box 1). At the upcoming conference, the Early Career Member Committee (ECMC) has organised four talks dedicated to "How to advance your career". This session will cover career tracks both in academia and in industry, and will provide information from an employer's and applicant's view: Gunilla WestergrenThorssen and Darcy Wagner will talk about a special tenure track system for junior research groups at Lund University (Lund, Sweden), which will be followed by David Wyatt and Mark Kästle from Boehringer Ingelheim (Ingelheim, Germany) sharing insights into industrial recruiting processes. The presentations will be followed by a panel discussion with all speakers and a networking event. Both give ECMs the perfect opportunity to learn a lot, get input on your own career path and possibly establish networks beneficial for your next position.

In order to offer ECMs a first opportunity to get involved in ERS, we will invite motivated people to contribute to our highlight article summarising the great science discussed in Estoril [1, 2]. Furthermore, we plan to assign half of the session chair positions to motivated ECMs. Thus, if you
Cite as: De Brandt J, Bartel S. Opportunities for early career members at the Lung Science Conference 2019. Breathe 2018; 14: 317-318.

@ERSpublications

The @ERStalk \#LSC2019 in Estoril, Portugal, provides early career members with various opportunities to get actively involved in the ERS and to strengthen their network http://ow.ly/nOPC30lp2V3 


\section{Box 1 ECM delegates session: How to advance your career}

Chairs: S. Bartel and P. Hiemstra

\begin{tabular}{|c|c|c|}
\hline Time & Presentation & Speaker \\
\hline 14:50-14:55 & Introduction & S. Bartel \\
\hline $14: 55-15: 10$ & $\begin{array}{l}\text { Tenure tracks and junior research groups: the Lund } \\
\text { University model }\end{array}$ & G. Westergren-Thorssen \\
\hline $15: 10-15: 25$ & Experiences from a junior research group leader & D. Wagner \\
\hline $15: 25-15: 40$ & Discussion with both speakers & \\
\hline $15: 40-15: 55$ & Successful entry paths into industry & D. Wyatt \\
\hline $15: 55-16: 10$ & Tips from a successful industry applicant & M. Kästle \\
\hline $16: 10-16: 25$ & Discussion with both speakers & \\
\hline $16: 25-16: 50$ & Panel discussion and networking event & \\
\hline
\end{tabular}

already know you will attend the LSC and would like to contribute in any way, please contact Sabine Bartel (sbartel@fz-borstel.de).

Finally, several members of the ECMC will also be present at the LSC, enabling you to get in contact with us and to become more actively involved in the ERS. We are looking forward to meeting you in the lovely Estoril.

\section{Conflict of interest}

J. De Brandt has nothing to disclose. S. Bartel reports receiving grants and personal fees from Bencard Allergie $\mathrm{GmbH}$ outside the submitted work.

\section{References}

1. Almendros I, Alsafadi HN, Bölükbas D, et al. Early Career Members at the ERS Lung Science Conference: cell-matrix interactions in lung disease and regeneration. Breathe 2018; 14: e78-e83.

2. Almendros I, Bartel S, Bostantzoglou C, et al. Early Career Members at the ERS LSC 2017: mechanistic overlap between chronic lung injury and cancer. Breathe 2017; 13 : 323-326. 